\title{
Group Acceptance Sampling Plans for Life Tests Based on Half Normal Distribution
}

\author{
B. Srinivasa Rao ${ }^{1 *}$, CH. Srinivas Kumar ${ }^{2}$ and K. Rosaiah ${ }^{3}$ \\ ${ }^{1}$ Department of Mathematics \& Humanities, R.V.R \& J.C College of \\ Engineering, Chowdavaram, Guntur-522 019, Andhra Pradesh, India. \\ ${ }^{2}$ Department of Mathematics, K.L.University, Vaddeswaram, \\ Guntur District-522 502, Andhra Pradesh, India. \\ ${ }^{3}$ Department of Statistics, Acharya Nagarjuna University, \\ Guntur- 522 010, Andhra Pradesh, India. \\ "Corresponding Author: boyapatisrinu@yahoo.com
}

Received: 11, February 2014 / Revised: 28, October 2014 / Accepted: 24, November 2014 (C) IAppStat-SL 2013

\begin{abstract}
In this article, a group acceptance sampling plan is developed based on truncated lifetimes when the lifetime of an item follows a half normal distribution. For a given group size, the minimum number of groups and the acceptance number required are determined for specified consumer's risk and the test termination time. The values of operating characteristic function for various quality levels are calculated and the minimum ratios of the true average life to the specified life at given producer's risk are obtained. The results are illustrated by examples.
\end{abstract}

Keywords: half normal distribution, group acceptance sampling plans, consumer's risk, operating characteristic (OC) function, producer's risk, truncated life test.

\section{Introduction}

Reliability study plays a vital role in the quality control analysis. On the basis of this study, an experimenter can save his time and cost to reach a result whether to accept the submitted lot or to reject it. An acceptance sampling plan is a scheme that establishes the minimum sample size to be used for testing. This becomes particularly important if the quality of product is defined by its lifetime. Often, it is implicitly assumed when designing a sampling plan that only a single item is put in a tester. However, in practice testers accommodating a multiple number of items at a time are used because 
testing time and cost can be saved by testing items simultaneously. The items in a tester can be regarded as a group and the number of items in a group is called the group size. For such a type of test, the determination of the sample size is equivalent to determine the number of groups. This type of testers is frequently used in the case of sudden death testing. An acceptance sampling plan based on such groups of items is called a group acceptance sampling plan (GASP). If the GASP is used in conjunction with truncated life tests, it is called a GASP based on truncated life test assuming that the lifetime of product follows a certain probability distribution.

Studies regarding truncated life tests can be found in Epstein(1954), Gupta and Groll(1961), Gupta(1962), Fertig and Mann(1980), Kantam and Rosaiah(1998), Kantam(2001) et. al., Baklizi(2003), Wu and Tsai(2005), Rosaiah and Kantam(2005), Tsai and $\mathrm{Wu} u(2006)$, Balakrishnan et. al.(2007), Aslam(2007), Srinivasa Rao et. al.(2008), Aslam and Kantam(2008), Aslam et. al.(2009), Srinivasa Rao et. al.(2009), Srinivasa Rao (2009), Lio et. al.(2010) , Srinivasa Rao et al(2010), Srinivasa Rao(2011), Aslam et. al.(2011), Aslam et. al.(2011a), Ramaswamy and Anburajan (2012).

In this paper, we describe the proposed group acceptance sampling plan(GASP) based on truncated life tests when the lifetime of a product follows half normal distribution in Section 2 . Operating characteristic (OC) is given in section 3. Producer's risk is given in Section 4. Examples are provided for an illustration in Section 5. The article is closed with summary and conclusions in Section 6.

\section{The Group Acceptance Sampling Plans (GASP)}

Statistical methods dealing with the properties and applications of the half normal distribution have been extensively used in diverse areas of applications particularly when the data are left/right truncated. The half normal distribution occurs when sampling from a normally distributed population where the signs of the negative observations are lost or are not relevant. Generally in reliability studies, the life time of a product ranges in $(0,1)$, this study proceeds from the observation that if we have a variable which follows half normal distribution then its probability density function (pdf) is given by

$$
p(x)=\frac{2 \theta}{\pi} e^{-\frac{x^{2} \theta^{2}}{\pi}}, \quad x \geq 0
$$


Its cumulative distribution function (cdf) is

$$
P(x)=\operatorname{erf}\left(\frac{\theta x}{\sqrt{\pi}}\right), \quad x \geq 0
$$

Half normal distribution is an increasing failure rate (IFR) model which is most useful in reliability studies. Because of this IFR nature we are motivated to study this distribution. Assume that the life time of a product follows half normal distribution with $\sigma$ as scale parameter .Its cumulative distribution function $\mathrm{F}($.) is given by

$$
F(t)=\operatorname{erf}\left(\frac{\theta \frac{t}{\sigma}}{\sqrt{\pi}}\right), \quad t \geq 0, \sigma>0
$$

Given $0<\mathrm{q}<1$, the $100^{\text {th }}$ percentile is given by

$$
t_{q}=\sigma \frac{\sqrt{\pi}}{\theta} \operatorname{erf}^{-1}(q)
$$

Substituting $\sigma$ in the Equation 2.3 in the scaled form we get

$$
\begin{gathered}
F(t)=\operatorname{erf}\left(\frac{\theta\left(\frac{t}{t_{q}}\right) \frac{\sqrt{\pi}}{\theta} \operatorname{erf}^{-1}(q)}{\sqrt{\pi}}\right), \\
F(t)=\operatorname{erf}\left(\operatorname{serf}^{-1}(q)\right)
\end{gathered}
$$

Where $\delta=\frac{t}{t_{q}}$. When the distribution is symmetric it is clear that the mean and median are same. But when the distribution is skewed which means that the side of the tail is long through which one can expect the mean to lean towards that side of the distribution. By increasing the amount of skewness, we can make the mean become much bigger and bigger in which case the proportion of the population below the mean can be made unduly large. This is what is meant by saying that mean would not represent a center of the distribution since more than $80 \%$ of the population may be below the mean. 
But if one were to use the median, there is always only $50 \%$ of the population less than the median.

Since for our present skewed population the median is a more approximate average for decision making about the quality of the life than population mean, we take $q=0.50$. Let $\mu$ be the true value of the median of the lifetime distribution of a product and $\mu_{0}$ denote the specified median under the assumption that the life time of an item follows half normal distribution. Based on the failure data, we want to test the hypothesis $H_{0}: \mu \geq \mu_{0}$ against $H_{1}: \mu<\mu_{0}$. A lot is considered as good if $\mu \geq \mu_{0}$ and bad if $\mu<\mu_{0}$. This hypothesis is tested using the group acceptance sampling scheme as:

1. Select the number of groups $g$ and allocate predefined $r$ items to each group so that the sample size for a lot will be $n=g . r$.

2. Select the acceptance number $c$ for a group and the experiment time $t_{0}$.

3. Perform the experiment for the $g$ groups simultaneously and record the number of failures for each group.

4. Accept the lot if at most $c$ failures occur in each of all groups.

5. Terminate the experiment if more than $\mathrm{c}$ failures occur in any group and reject the lot.

We are interested in determining the number of groups $g$ required for in the case of the half logistic distribution and various values of acceptance number $c$, whereas the group size $r$ and the termination time $t_{0}$ are assumed to be given. Since it is convenient to set the termination time as a multiple of the specified value $\mu_{0}$ of the median, we will consider $t_{0}=\delta \mu_{0}$ for a given constant (termination ratio). The probability $(\alpha)$ of rejecting a good lot is called the producer's risk, whereas the probability $(\beta)$ of accepting a bad lot is known as the consumer's risk. The parameter value $g$ of the proposed sampling plan is determined for ensuring the consumer's risk $\beta$. Often, the consumer's risk $\beta$ is expressed by the consumer's confidence level. If the confidence level is $p^{*}$, then the consumer's risk will be $\beta=1-p^{*}$. We will determine the number of groups $g$ in the proposed sampling plan so that the consumer's risk does not exceed a given value $\beta$. If the lot size is large enough, we can use the binomial distribution to develop the GASP. 
According to GASP the lot of products is accepted only if there are at most $c$ failures observed in each of the $g$ groups. So, the lot acceptance probability is given by

$$
\left(\sum_{i=0}^{c}\left(\begin{array}{l}
r \\
i
\end{array}\right) p_{0}^{i}\left(1-p_{0}\right)^{r-i}\right)^{g} \leq \beta
$$

Where $p_{0}=F_{t}\left(\delta_{0}\right)$ is the probability of a failure during the time $t=\delta t_{q}^{0}$. To save space, only the results of small sample sizes for $\beta=0.25,0.10,0.05,0.01 ; \quad r=2(1) 7 ; \quad c=0(1) 5$;

$\delta=0.7,0.8,1.0,1.2,1.5,2.0$ are displayed in Table 1 .

Table 1. Minimum number of groups ( $\mathrm{g}$ ) for the proposed plan in the case of half normal distribution

\begin{tabular}{|c|c|c|c|c|c|c|c|c|}
\hline$\beta$ & $\mathrm{r}$ & $\mathrm{c}$ & \multicolumn{6}{|c|}{$\delta$} \\
\hline & & & 0.7 & 0.8 & 1.0 & 1.2 & 1.5 & 2.0 \\
\hline 0.25 & 2 & 0 & 2 & 2 & 1 & 1 & 1 & 1 \\
0.25 & 3 & 1 & 4 & 4 & 2 & 2 & 1 & 1 \\
0.25 & 4 & 2 & 10 & 7 & 4 & 3 & 2 & 1 \\
0.25 & 5 & 3 & 22 & 14 & 7 & 4 & 3 & 2 \\
0.25 & 6 & 4 & 52 & 30 & 12 & 7 & 3 & 2 \\
0.25 & 7 & 5 & 125 & 64 & 22 & 10 & 4 & 2 \\
\hline 0.10 & 4 & 0 & 2 & 2 & 1 & 1 & 1 & 1 \\
0.10 & 5 & 1 & 3 & 3 & 2 & 2 & 1 & 1 \\
0.10 & 6 & 2 & 5 & 4 & 3 & 2 & 1 & 1 \\
0.10 & 7 & 3 & 10 & 7 & 4 & 3 & 2 & 1 \\
0.10 & 8 & 4 & 18 & 11 & 6 & 3 & 2 & 1 \\
0.10 & 9 & 5 & 35 & 20 & 8 & 5 & 2 & 1 \\
\hline 0.05 & 5 & 0 & 2 & 2 & 1 & 1 & 1 & 1 \\
0.05 & 6 & 1 & 3 & 2 & 2 & 2 & 1 & 1 \\
0.05 & 7 & 2 & 5 & 4 & 3 & 2 & 1 & 1 \\
0.05 & 8 & 3 & 8 & 6 & 3 & 2 & 2 & 1 \\
0.05 & 9 & 4 & 14 & 9 & 5 & 3 & 2 & 1 \\
0.05 & 10 & 5 & 26 & 15 & 7 & 4 & 2 & 1 \\
\hline 0.01 & 7 & 0 & 2 & 2 & 1 & 1 & 1 & 1 \\
0.01 & 8 & 1 & 3 & 2 & 2 & 2 & 1 & 1 \\
0.01 & 9 & 2 & 4 & 3 & 2 & 2 & 1 & 1 \\
0.01 & 10 & 3 & 7 & 5 & 3 & 2 & 2 & 1 \\
0.01 & 11 & 4 & 11 & 7 & 4 & 3 & 2 & 1 \\
0.01 & 12 & 5 & 17 & 11 & 5 & 3 & 2 & 1 \\
\hline
\end{tabular}




\section{Operating characteristic of the sampling plan}

The probability of acceptance can be regarded as a function of the deviation of the specified value $\mu_{0}$ of the median from its true value $\mu$. This function is called operating characteristic (OC) function of the sampling plan. Once the minimum number of groups $g$ is obtained, one may be interested to find the probability of acceptance of a lot when the quality is considered to be good if $\mu \geq \mu_{0}$ or $\frac{\mu}{\mu_{0}}$. The $\mathrm{OC}$ is given by

$$
L(P)=\left(\sum_{i=0}^{c}\left(\begin{array}{l}
r \\
i
\end{array}\right) p_{0}^{i}\left(1-p_{0}\right)^{r-i}\right)^{g}
$$

Using Equation 3.1 the $\mathrm{OC}$ values can be obtained for any sampling plan. To save space we present the OC values for sampling plans with

$$
\begin{aligned}
& \frac{\mu}{\mu_{0}}=2,4,6,8,10,12 ; \beta=0.25,0.10,0.05,0.01 \\
& \delta=0.7,0.8,1.0,1.2,1.5,2.0 ; \quad r=4,6,7,9 ; \text { are given in table } 2 .
\end{aligned}
$$

Table 2. Operating characteristic values of the group sampling plan for half normal distribution

\begin{tabular}{|c|c|c|c|c|c|c|c|c|c|}
\hline$\beta$ & $\mathrm{r}$ & $\mathrm{g}$ & $\delta$ & \multicolumn{6}{|c|}{$\frac{\mu}{\mu_{0}}$} \\
\hline & & & & 2 & 4 & 6 & 8 & 10 & 12 \\
\hline 0.25 & 4 & 9 & 0.7 & 0.8114 & 0.9725 & 0.9916 & 0.9964 & 0.9981 & 0.9989 \\
0.25 & 4 & 7 & 0.8 & 0.8070 & 0.9715 & 0.9912 & 0.9962 & 0.9981 & 0.9989 \\
0.25 & 4 & 4 & 1.0 & 0.8118 & 0.9720 & 0.9913 & 0.9963 & 0.9981 & 0.9989 \\
0.25 & 4 & 3 & 1.2 & 0.7866 & 0.9675 & 0.9899 & 0.9956 & 0.9977 & 0.9987 \\
0.25 & 4 & 2 & 1.5 & 0.7727 & 0.9648 & 0.9890 & 0.9952 & 0.9975 & 0.9986 \\
0.25 & 4 & 1 & 2.0 & 0.8075 & 0.9706 & 0.9908 & 0.9960 & 0.9979 & 0.9988 \\
\hline 0.10 & 6 & 5 & 0.7 & 0.6680 & 0.9408 & 0.9809 & 0.9916 & 0.9956 & 0.9974 \\
0.10 & 6 & 4 & 0.8 & 0.6322 & 0.9317 & 0.9776 & 0.9901 & 0.9948 & 0.9969 \\
0.10 & 6 & 3 & 1.0 & 0.5730 & 0.9151 & 0.9716 & 0.9874 & 0.9933 & 0.9961 \\
0.10 & 6 & 2 & 1.2 & 0.5794 & 0.9147 & 0.9711 & 0.9870 & 0.9931 & 0.9959 \\
0.10 & 6 & 1 & 1.5 & 0.6589 & 0.9324 & 0.9769 & 0.9896 & 0.9944 & 0.9967 \\
0.10 & 6 & 1 & 2.0 & 0.5209 & 0.8941 & 0.9627 & 0.9829 & 0.9908 & 0.9945 \\
\hline 0.05 & 7 & 5 & 0.7 & 0.5343 & 0.9049 & 0.9682 & 0.9858 & 0.9925 & 0.9956 \\
0.05 & 7 & 4 & 0.8 & 0.4951 & 0.8913 & 0.9630 & 0.9834 & 0.9912 & 0.9948 \\
0.05 & 7 & 3 & 1.0 & 0.4328 & 0.8674 & 0.9537 & 0.9790 & 0.9888 & 0.9933 \\
0.05 & 7 & 2 & 1.2 & 0.4460 & 0.8682 & 0.9533 & 0.9786 & 0.9885 & 0.9931 \\
0.05 & 7 & 1 & 1.5 & 0.5457 & 0.8965 & 0.9630 & 0.9829 & 0.9908 & 0.9945 \\
0.05 & 7 & 1 & 2.0 & 0.3955 & 0.8421 & 0.9413 & 0.9723 & 0.9849 & 0.9909 \\
\hline 0.01 & 9 & 4 & 0.7 & 0.3810 & 0.8445 & 0.9447 & 0.9747 & 0.9864 & 0.9919 \\
0.01 & 9 & 3 & 0.8 & 0.3705 & 0.8356 & 0.9405 & 0.9735 & 0.9852 & 0.9911 \\
0.01 & 9 & 2 & 1.0 & 0.3608 & 0.8247 & 0.9348 & 0.9694 & 0.9833 & 0.9900 \\
0.01 & 9 & 2 & 1.2 & 0.2377 & 0.7549 & 0.9045 & 0.9543 & 0.9748 & 0.9847 \\
0.01 & 9 & 1 & 1.5 & 0.3510 & 0.8089 & 0.9254 & 0.9640 & 0.9800 & 0.9878 \\
0.01 & 9 & 1 & 2.0 & 0.2111 & 0.7232 & 0.8856 & 0.9432 & 0.9680 & 0.9800 \\
\hline
\end{tabular}




\section{Producer's Risk}

The producer may be interested in enhancing the quality level of the product so that the acceptance probability should be larger than a specified level. For a given value of the producer's risk, say $\gamma$, the minimum ratio can be obtained by satisfying the following inequality

$$
\left(\sum_{i=0}^{c}\left(\begin{array}{l}
r \\
i
\end{array}\right) p_{0}^{i}\left(1-p_{0}\right)^{r-i}\right)^{g} \leq 1-\gamma
$$

To save space, the minimum values of the ratio $\frac{\mu}{\mu_{0}}=2$ in case of half normal distribution based on the values given in table 1 for the acceptability of a lot at the producer's risk of $\gamma=0.05$ are presented in table 3 .

Table 3. Minimum ratio of the values of true median and the specified median for the producer's risk of $\gamma=0.05$ in the case of half normal distribution.

\begin{tabular}{|c|c|c|c|c|c|c|c|c|}
\hline$\beta$ & $\mathrm{r}$ & $\mathrm{c}$ & \multicolumn{7}{|c|}{$\delta$} \\
\hline & & & 0.7 & 0.8 & 1.0 & 1.2 & 1.5 & 2.0 \\
\hline 0.25 & 2 & 0 & 29.5580 & 33.7805 & 21.2456 & 25.4947 & 31.8686 & 42.4911 \\
0.25 & 3 & 1 & 5.6427 & 6.4488 & 5.6548 & 6.7857 & 5.9340 & 7.9120 \\
0.25 & 4 & 2 & 3.3588 & 3.3929 & 3.4892 & 3.7843 & 4.0972 & 4.2572 \\
0.25 & 5 & 3 & 2.4671 & 2.5037 & 2.6031 & 2.6860 & 2.7746 & 3.0410 \\
0.25 & 6 & 4 & 2.0645 & 2.0999 & 2.1554 & 2.2984 & 2.3759 & 2.8857 \\
0.25 & 7 & 5 & 1.8306 & 1.8577 & 1.9147 & 1.9857 & 2.0841 & 2.4226 \\
\hline 0.10 & 4 & 0 & 58.9289 & 67.3473 & 42.2257 & 50.6708 & 63.3385 & 84.4513 \\
0.10 & 5 & 1 & 8.7541 & 10.0046 & 10.1248 & 12.1498 & 10.5419 & 14.0558 \\
0.10 & 6 & 2 & 4.4043 & 4.6469 & 5.2363 & 5.4204 & 5.2368 & 6.9824 \\
0.10 & 7 & 3 & 3.1683 & 3.2841 & 3.5145 & 3.8890 & 4.3296 & 4.7113 \\
0.10 & 8 & 4 & 2.4976 & 2.5583 & 2.7880 & 2.8486 & 3.2333 & 3.6368 \\
0.10 & 9 & 5 & 2.1402 & 2.2031 & 2.3112 & 2.5290 & 2.6264 & 3.0789 \\
\hline 0.05 & 5 & 0 & 73.6142 & 84.1305 & 52.7154 & 63.2584 & 79.0730 & 105.4307 \\
0.05 & 6 & 1 & 10.6817 & 9.8752 & 12.3439 & 14.8127 & 12.8278 & 17.1037 \\
0.05 & 7 & 2 & 5.2734 & 5.5610 & 6.2619 & 6.4748 & 6.2408 & 8.3211 \\
0.05 & 8 & 3 & 3.5151 & 3.7091 & 3.8127 & 4.0701 & 5.0876 & 5.5234 \\
0.05 & 9 & 4 & 2.7519 & 2.8463 & 3.1080 & 3.3071 & 3.7496 & 4.2088 \\
0.05 & 10 & 5 & 2.3355 & 2.4040 & 2.5909 & 2.7798 & 3.0112 & 3.4573 \\
\hline 0.01 & 7 & 0 & 102.9848 & 117.6969 & 73.6945 & 88.4334 & 110.5418 & 147.3890 \\
0.01 & 8 & 1 & 14.5283 & 13.4173 & 16.7717 & 20.1260 & 17.3878 & 23.1837 \\
0.01 & 9 & 2 & 6.4571 & 6.6414 & 7.1427 & 8.5713 & 8.2360 & 10.9813 \\
0.01 & 10 & 3 & 4.4068 & 4.5805 & 4.9456 & 5.2710 & 6.5887 & 7.1300 \\
0.01 & 11 & 4 & 3.3337 & 3.4326 & 3.7609 & 4.2118 & 4.7677 & 5.3350 \\
0.01 & 12 & 5 & 2.7161 & 2.8513 & 3.0439 & 3.2870 & 3.7703 & 4.3164 \\
\hline & & & & & & & & \\
\end{tabular}




\section{Tables and Examples}

The design parameters of GASP are found at various values of the consumer's risk and the test termination time multiplier in Table 1. It should be noted that if one needs the minimum sample size, it can be obtained by $\mathrm{n}=$ $\mathrm{r} \times \mathrm{g}$. Table 1 indicates that, as the test termination time multiplier $\delta$ increases, the number of groups $g$ decreases, i.e., a smaller number of groups is needed, if the test termination time multiplier increases at a fixed group size. For an example, from Table 1 , if $\beta=0.01, r=6, c=2$ and a change from 0.7 to 0.8 , the required values of the design parameters of GASP change from $g=5$ to $g=4$. However, this trend is not monotonic since it depends on the acceptance number as well. The probability of acceptance for the lot at the median ratio corresponding to the producer's risk is given in Table 2. Finally, Table 3 presents the minimum ratios of true median to the specified median for the acceptance of a lot with producer's risk $\gamma=0.05$ for given parameter values.

Suppose that the lifetime of a product follows the half normal distribution, it is desired to design a GASP to test if the median is greater than 1,000 hours based on a testing time of 700 hours and using testers equipped with 6 items each. It is assumed that $c=2$ and $\beta=0.10$. This gives the termination multiplier $\delta=0.700$. From Table 1 the minimum number of groups required is obtained as $g=5$. Thus, we will draw a random sample of size $n=30$ items and allocate 6 items to each of the 5 groups to put on test for 700 hours. This indicates that a total of 30 products are needed and that 6 items are allocated to each of the 5 testers. We will accept the lot if no more than 2 failures occur before 700 hours in each of 5 groups. We terminate the experiment as soon as the $3^{\text {rd }}$ failure occurs before the $700^{\text {th }}$ hours. For this proposed sampling plan the probability of acceptance is $p=0.9408$ when the true value of the median is $\mu=4,000$ hours. This shows that, if the true value of the median is 4 times of the required value $\mu_{0}=1000$ hours, the producer's risk is $\alpha=0.0592$. If we need the ratio to assure a producer's risk of $\gamma=0.05$, we can obtain it from Table 3. For example, when $\beta=0.10, r=6, g=5, c=2$ and $\delta=0.700$, the required ratio is $\frac{\mu}{\mu_{0}}=4.4043$. 


\section{Summary and Conclusions}

In this paper, a group acceptance sampling plan from a truncated life test is proposed in the case of half normal distribution. The number of groups and the acceptance number are determined when the consumer's risk $(\beta)$ and the other plan parameters are specified. It is observed that the minimum number of groups required decreases as the test termination time multiplier increases. Moreover, the operating characteristics function increases disproportionately when the quality improves. This GASP can be used when a multiple number of items are tested simultaneously. Clearly, such a tester would be beneficial in terms of test time and test cost.

\section{Acknowledgments}

The authors thank the editor, reviewers and Prof. N. Balakrishnan for their helpful suggestions, comments and encouragement, which helped in improving the final version of the paper.

\section{References}

1. Aslam.M (2007) Double acceptance sampling based on truncated life tests in Rayleigh distribution European Journal of Scientific Research, 17:605-611, 2007

2. Aslam.M.,and Kantam.R.R.L.(2008) Economic reliability acceptance sampling plan based on truncated life tests in Birnbaum-Saunders distribution Pakistan Journal of Statistics, 24:269-276, 2008

3. Aslam.M.Jun.C.H., and Ahmad.M. (2009) A group sampling plan based on truncated life tests for gamma distribution Pakistan Journal of Statistics, 25:333-340, 2009

4. Aslam.M.,Debasis Kundu., Jun.C.H., and Ahmad.M. (2011) Time truncated group acceptance sampling plans for generalized exponential distribution Journal of Testing and Evaluation, 39(4): 968-976. 2011

5. Aslam.M.,Jun.C.H., Ahmad.M., and Rasool.M. (2011) Group accepatnce sampling plans for resubmitted lots under Burr-type XII distributions Chinese Intuition of Industrial Engineers, 28(8):606-615,2011

6. Baklizi.A. (2003) Acceptance sampling based on truncated life tests in the pareto distribution of the second kind Advances and Applications in Statistics, 3:33-48, 2003 
7. Balakrishnan.,N., Leiva.V., and Lopez.J. (2007) Acceptance sampling plans from truncated life tests based on generalized Birnbaum-saunders distribution Communications in Statistics-Simulation and Computation, 36:643-656, 2007. DOI: 10.1080/03610910701207819

8. Epstein.B. (1954) Truncated life tests in the exponential case Annals of Mathematical Statistics,25:555-564, 1954.

DOI: $10.1214 / \mathrm{aoms} / 1177728723$

9. Fertig.F.W., and Mann.N.R.(1980) Life-test sampling plans for twoparameter Weibull populations. Technometrics,22:165-177.

DOI: 10.1080/00401706.1980.10486131

10. Gupta.S.S., and Groll.P.A. (1961) Gamma distribution in acceptance sampling based on life tests. Journal of the American Statistical Association, 56:942-970, DOI: 10.1080/01621459.1961.10482137

11. Gupta.S.S. (1962) Life test sampling plans for normal and lognormal distribution. Technometrics, $4: 151-175$,

DOI: $10.1080 / 00401706.1962 .10490002$

12. Kantam.R.R.L., and Rosaiah.K. (1998) half logistic distribution in acceptance sampling based on life tests IAPQR Transactions, 23(2): $117\{125$.

13. Kantam.R.R.L. (2001) Srinivasa Rao.G., and Rosaiah.K., Acceptance sampling based on life tests: Log-logistic distribution Journal of Applied Statistics, 28(1):121-128, DOI: 10.1080/02664760120011644

14. Lio.Y.L., Tsai.T.R., and Wu.S.J. (2010) Acceptance sampling plans from truncated life tests based on Birnbaum-Saunders distribution for percentiles. Communications in Statistics-Simulation and Computation,39:119-136.

15. Ramaswamy.A.R.S., and Anburajan.P. (2012) Group acceptance sampling plans using weighted binomial on truncated life tests for inverse Rayleigh and Log-logistic distributions IOSR Journal of Mathematics, 2(3): 33-38.

16. Rosaiah.K., and Kantam.R.R.L. (2005) Acceptance sampling plans based on Inverse Rayleigh Distribution Economic Quality Control, 20(2): 277286.

17. Srinivasa Rao.G., Ghitany.M.E., and Kantam.R.R.L. (2008) Acceptance sampling plans for Marshall-Olkin extended Lomax Distribution . International Journal of Applied Mathematics, 21:315-325. 
18. Srinivasa Rao.G., Ghitany.M.E., and Kantam.R.R.L. (2009) Reliability test plans for Marshall-Olkin extended exponential distribution. Applied mathematical sciences, 3(55): 2745-2755.

19. Srinivasa Rao.G. (2009) A group acceptance sampling plans for truncated life tests for Marshall- Olkin extended Lomax distribution. Electronic Journal of Applied Statistical Analysis , 3(1):18-28.

20. Srinivasa Rao.G.,and Kantam.R.R.L. (2010) Acceptance sampling plans from truncated life tests based on log-logistic distribution for percentiles Economic Quality Control, 25(2):153-167.

21. Srinivasa Rao.G. (2011) A hybrid group acceptance sampling plans for lifetimes based on log-logistic distribution Journal of Reliability and Statistical Studies, 4(1):31-40.

22. Tsai.T.R., andWu.S.J. (2006) Acceptance sampling plans based on truncated life test for generalized Rayleigh distribution. Journal of Applied Statistics, 33:595-600, DOI: 10.1080/02664760600679700

23. Wu.C.J., and Tsai.T.R. (2005) Acceptance sampling plans for BirnbaurnSaunders distribution under truncated life tests. International Journal of Reliability, Quality and Safety Engineering,12:507-519.

DOI: $10.1142 / \mathrm{S} 0218539305001987$ 